\title{
De la historia natural
} a la biología moderna: máquinas, robots y cabinas de vuelo en el Natural History Museum (NHM, Museo de Historia Natural), Londres, Reino Unido

From Natural History to Modern Biology: Machines, Robots and Flight Decks in the Natural History Museum (NHM), London, United Kingdom

\author{
Gustavo Corral Guillé \\ Investigador independiente, México \\ gustavo.corral@gmail.com
}

\section{Resumen}

El presente ENSAYO ofrece una mirada al Natural History Museum (NHM, Museo de Historia Natural), Londres, Reino Unido, entre finales de la década de 1960 y principios de la de 1980 con la finalidad de explorar la forma en que entonces cambió tanto su identidad institucional como su política expositiva. Intento mostrar que, con la idea de modificar su imagen, el modelo de discurso museográfico del NHM dejó de centrarse en su colección —esto es, los especímenes de historia natural- para consagrarse a los visitantes y prestar especial atención al nuevo enfoque de la biología contemporánea. Para ello se plantea que Human Biology, primera exposición del Nuevo Programa de Exposiciones (NPE) ${ }^{1}$ del NHM, se diseñó en concordancia con la tendencia reduccionista que caracterizaba a la ciencia contemporánea, estructurada en torno de los sistemas de información. Gracias a esta orientación, Human Biology se convirtió en un espacio de producción y legitimación de una particular perspectiva científica, muy cercana a la enarbolada por los asesores externos que colaboraron en la especificación de sus contenidos museográficos, desplegados, así, a partir de metáforas visuales y estrategias interactivas.

\section{Palabras clave}

museos de historia natural; Londres; comunicación de la ciencia; metáforas visuales; interactividad

\section{Abstract}

This ESSAY provides an overview of the Natural History Museum (NHM) in London, United Kingdom, between the late 1960s and early 1980s, in order to explore how it changed both its institutional identity as well as its exhibition policy during this period. It intends to demonstrate that, in

\footnotetext{
${ }^{1}$ Este nuevo Programa de Exposiciones se conoció en el NHM como New Exhibition Scheme [NES].
} 
order to alter its image, the museographic discourse of the NHM stopped focusing on its collection - that is, the natural history specimens - to instead concentrate on the visitors and pay particular attention to the new approach of contemporary biology. To this end, it is posed that $\mathrm{Hu}$ man Biology, the first exhibition of the New Exhibition Program (NEP), was designed in accordance with the reductionist tendencies that characterized contemporary science, structured around the information systems. Hence, through visual metaphors and interactive strategies, Human Biology became a production and legitimization space for a particular scientific point of view, which was very close to that exposed by the external advisers who collaborated in the specification of its museographic contents.

\section{Key words}

Natural History Museum; London; science communication; visual metaphors; interactivity

\section{Introducción}

os museos como forma institucional tienen una carga ideológica que les concede el papel de depositarios de conocimiento y significados valiosos para la sociedad (Gray 2015), esto es un valor simbólico que, de acuerdo con Bloom (1992:19), "es el resultado de innumerables decisiones" en relación con los contenidos de las exposiciones, la información elegida para presentarlas y lo que los creadores optan por mostrar o silenciar. La práctica expositiva no puede entenderse como un ejercicio neutral, objetivo y apolítico: por el contrario, se trata de una tarea en la que se entremezclan elementos como los hechos y los valores, la ciencia y la política, el conocimiento y el poder (Vergo 1989; Bloom 1992; Arnold 1996; Macdonald 1998:1-2).

El caso de los museos de ciencia y tecnología no es la excepción. Tal y como lo demuestran Macdonald (1998) y Pedretti (2002) — dos importantes textos de la nueva museología especializados en museos de ciencia-, en ellos se construye tanto un tipo particular de ciencia para el público, al que se le señala cómo debe pensar sobre un tema en concreto, como consensos políticos para conferirle legitimidad a dicha perspectiva. Para estudiar más minuciosamente esa afirmación, analizaré una exposición inaugurada en el Natural History Museum (NHM, Museo de Historia Natural), de Londres, en 1977. Este ENSAYO se desprende de la tesis de doctorado (Corral 2015) intitulada "Museo de Historia Natural de Londres, 1968-1981. Una perspectiva histórica", presentada en la Universitat Autònoma de Barcelona (UBA, España). ${ }^{2}$ Sin embargo, la presente contribución se centra en la primera de las cinco exposiciones que analicé en mi tesis, lo que me

\footnotetext{
${ }^{2}$ Como resultado de esa investigación doctoral, también se publicó el artículo "El Museo de Historia Natural de Londres y su nuevo programa de exposiciones [NPE] en la década de 1970. Perfilando nuevos objetivos educativos" (Corral 2016).
}

permite ofrecer información novedosa sobre el cambio de discurso expositivo que experimentó el NHM desde aquel momento. El ENSAYO profundiza, además, en el papel que desempeñaron las metáforas visuales y la interactividad en el mencionado cambio en las políticas expositivas.

Human Biology-An Exhibition of Ourselves fue esa primera exposición del NPE del NHM a la que me refiero, la cual comenzó a perfilarse en 1968 con el nombramiento de Frank Claringbull como director del museo. ${ }^{3}$ Los actores involucrados en el desarrollo de la muestra crearon una retórica visual y narrativa de las ideas de renovación de los contenidos del NHM que tenían en mente (Corral 2015). Se trataba de un grupo muy heterogéneo de nuevos profesionales del museo, entre los que destacaban los denominados transformadores, esto es, parejas formadas por un científico y un diseñador industrial. Human Biology contó con seis de ellas, encargadas de conceder la misma importancia a los contenidos científicos que a su representación visual. Estos transformadores trabajaban en equipo con otros grupos profesionales, como los editores y los escritores científicos, pues Claringbull sostenía que los científicos no eran capaces de escribir de manera accesible para el público las etiquetas y los paneles. Había también diseñadores gráficos e ilustradores, más los fabricantes de los modelos y los dispositivos interactivos.

Como se verá adelante, el grupo de asesores científicos externos al NHM que debían evaluar las propuestas de los transformadores y ofrecer sugerencias sobre el tema resultaron determinantes para el enfoque elegido para la exposición y el significado de algunas de las estrategias y técnicas expositivas. Estos asesores fueron el zoólogo y neurofisiólogo John Zachary Young, el especialista en salud infantil y crecimiento James Mourilyan Tanner, el psicólogo Aimable Robert Jonckheere y el ingeniero eléctrico William Kenelm Taylor, todos, profesores del University College London. Participaron, asimismo como asesores, Angela Hobsbaum y John Versey, profesores de desarrollo infantil en el Departamento de Desarrollo Infantil y Educación Primaria del Instituto de Educación de Londres, y Richard Langton Gregory, experto en psicología perceptual de la Universidad de Bristol.

\footnotetext{
3 Frank Claringbull (1911-1990) estudió en el Queen Mary College de Londres (actualmente, Queen Mary University of London [QMUL], Reino Unido), donde se graduó como licenciado en química en 1932 y obtuvo el grado de doctor en 1935, año en el que se unió al personal del Departamento de Mineralogía del Natural History Museum (NHM). De 1939 a 1945 se separó del museo y se trasladó a la Universidad de Birmingham para aprender las técnicas de cristalografía de rayos $X$ utilizadas para la determinación de estructuras cristalinas. En 1945 regresó a trabajar al museo y describió aun hasta siete minerales nuevos, incluidas la sinhalita, la taaffeita y la kalsilita. Gracias a su trabajo, en 1953 se lo nombró jefe del Departamento de Mineralogía, cargo que desempeñaría hasta 1968, año en que asumió la dirección del NHM. En ese periodo publicó, junto con Lawrence Bragg, la obra Crystal Structures of Minerals, sobre su amplia investigación mineralógica (cfr. Claringbull y Bragg 1965).
} 
En concreto, los contenidos y el estilo de Human Biology proyectaban el deseo de dichos actores por homologar la "historia natural con las ciencias biológicas modernas" (Claringbull 1974:1). En lugar de resaltar la diversidad de la naturaleza y su estudio morfológico, como se hacía desde el enfoque tradicional, la exposición ofreció uno más abstracto y funcional, con importantes influencias de los sistemas cibernéticos y las tecnologías de información. ${ }^{4}$ Por lo tanto, del análisis de esa exposición se desprenden aquí tres intenciones.

Primero, demostrar que la exposición fue el punto de partida para echar a andar las estrategias del NPE ideadas para modificar la identidad, la misión y las prácticas del museo.

Segundo, al examinar Human Biology con ayuda de material de archivo y entrevistas recientes que explican su proceso de creación, reconocer los intereses y la lógica institucional que provocaron ese cambio de enfoque museográfico en el NHM de la historia natural a la "biología moderna".

Tercero, desde una perspectiva histórica, comprender cómo esos cambios proporcionan una muestra de que los museos son espacios en los que se intersecan la política, la cultura y la ciencia.

\section{Human Biology: la transformación de la identidad y las prácticas expositivas del NHM}

En 1972, Frank Claringbull presidió un grupo de trabajo, integrado por miembros del personal científico del NHM, que evaluaría las políticas expositivas de la institución, el cual elaboró el documento "A proposal for a new approach to the visiting public", que Claringbull presentó a los Trustees en febrero de ese año (NHM 1972). ${ }^{5} \mathrm{El}$ texto señalaba las que, a su juicio, eran las principales deficiencias de la política expositiva: "es errónea porque su enfoque principal es la diversidad y omite buena parte de otros temas. Está conceptualmente fragmentada y es estática, no pone atención a los procesos e interacciones naturales y refleja la división del museo en cinco departamentos diferentes [Zoología, Botánica,

\footnotetext{
${ }^{4}$ Para mayor detalle sobre el enfoque expositivo tradicional del NHM véanse Cannon (1962), Stearn (1981), y Whitehead y Keates (1981).

${ }^{5}$ El Board of Trustees del NHM es responsable de supervisar la gestión del Museo, determinar las políticas a seguir y, junto con el personal, fijar la dirección de la institución. Estaba presidido en ese momento por Eric Smith, que antes había sido profesor de zoología en las universidades de Manchester, Sheffield y Cambridge, así como en el Queen Mary College (QMC), además, de vicepresidente de la Linnean Society of London (1954-1955) y de la Zoological Society of London (1959-1991), vicerrector del QMC (1963-1965) y miembro del Science Research Council de Gran Bretaña (1965-1967). Los otros miembros del Board of Trustees eran los botánicos Arthur Roy Clapham y Thomas Maxwell Harris, los naturalistas John David Gathorne-Hardy y Miriam Rothschild, los geólogos William Alexander Deer y Alwyn Williams, así como el funcionario público y administrador de museos Arthur Drew (NHM 1973).
}

Mineralogía, Entomología y Paleontología]" (Miles y Alt 1979:158-162).

El grupo sugería que para subsanar esas deficiencias había que establecer un nuevo programa de exposiciones que presentara "una visión moderna de la historia natural" de una forma que fuese comprensible para el público con pocos conocimientos (NHM 1972:1). Los Trustees aprobaron el documento y desde entonces comenzó el desarrollo del NPE, pensado para actualizar los contenidos de las galerías y las técnicas expositivas en aras de dar mayor prioridad a las misiones pedagógica y comunicativa del museo (Stearn 1981:365-374; Perks 2012; Corral 2015). Con ese propósito, el nuevo programa debía girar en torno de cuatro ejes temáticos: Ser humano, Ecología, Procesos vitales y comportamiento, y Evolución y diversidad (NHM 1972b). Éstos caracterizaban lo que el grupo entendía por "biología moderna", centrada en aspectos tan variados como "la forma, la estructura y el comportamiento de los seres vivos, de los factores químicos y físicos en su funcionamiento, de sus procesos de desarrollo, reproducción y herencia, de las relaciones entre ellos y con su entorno, así como de su historia" (NHM 1972:3).

En octubre de 1973, Roger Miles, paleontólogo especialista en peces que desarrollaba trabajos de investigación y tareas generales de curaduría, además de que había formado parte del grupo evaluador, fue elegido por Claringbull, gracias a su "don para el trabajo expositivo y educativo", para coordinar la planificación y el desarrollo del NPE (NHM Archive Catalogue s.f.). Miles debía impulsar por medio del NPE cambios trascendentes en la gestión museística y nuevas prácticas en la creación expositiva para que los visitantes consideraran el museo "no sólo como una fuente de información sino también como una fuente de recreación y entretenimiento" (NHM 1977b:6). Como tales, dichos cambios fueron parte de un movimiento más amplio que buscaba convertir los museos en sitios de esparcimiento, difuminando la frontera entre estas instituciones y los diversos espacios de ocio y disfrute del tiempo libre (Morris 2010; Rader y Cain 2014).

En 1974 comenzó la planificación de Human Biology-An Exhibition of Ourselves, la primera exposición del NPE que Claringbull y Miles veían como un proyecto piloto para medir la capacidad de su equipo y poner en práctica los fundamentos pedagógicos y comunicativos mencionados (Claringbull 1974:1; NHM 1975:2). El eventual éxito de esta exposición aumentaría la confianza de alcanzar el objeto propuesto por Frank Claringbull y Roger Miles para los siguientes 15 o 20 años del NHM: crear "un enfoque científico al problema de ayudar al visitante a entender la naturaleza" (NHM 1975:2). La exhibición proporcionaría experiencia y práctica en el diseño y la producción de lo que Miles denominó tecnología museística, un esquema de trabajo que permitiría crear de manera sistemática exposiciones didácticas (Miles y Tout 1979:209-224). 
El costo de Human Biology fue de 650000 libras aproximadamente, la mayor parte de las cuales provino del Department of the Environment (DOE, Departamento de Medio Ambiente, Reino Unido), ${ }^{6}$ luego de que el Advisory Board for the Research Councils (ABRC, Grupo Consultor para los Consejos de Investigación, Reino Unido) recomendara al gobierno británico la conveniencia de apoyar financieramente la renovación de las exposiciones del $\mathrm{NHM}{ }^{7}$ De hacerlo, opinaba el ABRC, esta institución incrementaría el interés de los jóvenes por la ciencia.

Inaugurada el 24 de mayo de 1977, Human Biology fue una exposición permanente, instalada en lo que hasta entonces había sido la Fish and Reptile Gallery (Galería de Peces y Reptiles) del NHM (Miles 1986:230). Ocupaba más de $1100 \mathrm{~m}^{2}$ en 14 pabellones que contaban con 161 piezas en exhibición, entre las que destacaban 28 dispositivos interactivos, 7 instalaciones totalmente audiovisuales y varios modelos tridimensionales (Miles 1986:230).

Esta exhibición introdujo una nueva cultura de curaduría en el NHM, al quitar el control de las galerías a los curadores y, en su lugar, dárselo a equipos de nuevos profesionales con habilidades comunicativas, pedagógicas y de diseño (Perks 2012; Corral 2015, 2016). Posteriormente, en enero de 1975, se formó el Department of Public Services (DPS, departamento de Servicios Públicos), encabezado por Roger Miles, con el fin de fusionar y centralizar las actividades de educación y exposición del museo. El nuevo departamento debía coordinar el trabajo del nuevo personal contratado para llevar a cabo el diseño expositivo, así como brindar los servicios educativos y evaluar tales exposiciones (Stearn 1981).

Human Biology puso en tela de juicio el valor de las colecciones como herramienta para el conocimiento. En opinión de Miles:

Las galerías públicas de principios de los años setenta no cumplían con el propósito educativo del museo ni representaban una exposición actualizada de la historia natural. No estaban al día sobre el desarrollo de la televisión y otros medios de comunicación y no reflejaban el papel del museo como fuente permanente y accesible de especímenes, información y asesoramiento sobre la historia natural moderna (Miles 1979:8, traducción del autor).

\footnotetext{
${ }^{6}$ Actualmente al DOE se lo conoce como Department for Environment, Foods and Rural Affairs (DEFRA, Ministerio del Medio Ambiente, Alimentación y Asuntos Rurales).

${ }^{7}$ Se trataba de una suma bastante importante para el montaje de una exposición, incluso comparado con el de exposiciones inmersas en programas de renovación de otros museos. Por ejemplo, la exposición Britain Before Man, gran apuesta del Geological Museum, inaugurada también en 1977, costó poco más de 100000 libras (Fifield 1977). El ABRC era el organismo encargado de asesorar al Ministerio de Educación y Ciencia sobre el total del presupuesto requerido para la ciencia y la forma en que debía distribuirse (Pearson 1981:129).
}

Al adoptar esta posición, Miles daba a entender que, en su proyecto de redefinición, la misión educativa del NHM dependía de encontrar para éste una nueva identidad, es decir, una definición actualizada acerca de lo que debía ser y lo que debía hacer. El nuevo enfoque centrado en el visitante, dedicado a la educación y el entretenimiento, debería ser comparable y ofrecer un potencial similar al de los medios de comunicación. Desde su perspectiva, el uso de herramientas comunicativas en las nuevas exposiciones, como los audiovisuales y, como se verá más adelante, los dispositivos interactivos, mejoraría el paisaje monótono y estático del museo tradicional en el que los especímenes eran la única estrategia pedagógica.

Por otra parte, la transferencia total de responsabilidades en la autoría de las exposiciones de los curadores a los miembros del Department of Public Services, aquí denominados científicos-transformadores, determinó el rumbo definitivo de la política expositiva, ya que los últimos no sólo cambiaron la información del NHM sino también su cultura curatorial, y profesionalizaron su práctica mediante un proceso de especialización y división de tareas. Así, se estableció que los científicostransformadores a cargo de especificar los contenidos tendrían que expresar interés en la comunicación de la ciencia, aunque no tuvieran experiencia en los temas en cuestión (Perks 2012:94). Por ejemplo, de los seis miembros del equipo involucrados en la generación de la información que se mostraría en Human Biology, cuatro trabajaban en el NHM: Brian Rosen, con experiencia en geología; Theya Molleson, en antropología, y Richard Vane-Wright y Richard Lane, en entomología; los otros dos, Brenda Winch y Steve Parker, eran científicos externos con amplia experiencia en la comunicación de la ciencia (Corral 2015:118-123).

Estos cambios en la identidad y las prácticas expositivas tradicionales suscitaron controversias internas que incluso trascendieron los límites del NHM. Por un lado, los estudios de visitantes indicaron que con la nueva exposición el museo alcanzó un éxito cuantitativo por la cantidad de público que atrajo: más de 30000000 personas en 1977, con lo que Human Biology fue la exposición permanente más popular que el NHM haya producido hasta entonces (Alt 1980). Por otro lado, algunos profesionales del mundo de los museos, escépticos sobre estos cambios, recibieron con consternación la nueva exposición, pues su contenido ya no reflejaba las fortalezas que proporcionaban las colecciones: a los objetos ya no se los consideraba como piezas de una colección tradicional, sino sólo como ilustraciones de la historia construida en el guion de la exposición (Greenaway 1983; Woolley 2014). El problema, argüían estos críticos, era que, al supeditar las colecciones a una historia, o narrativa, el visitante quedaba imposibilitado de hacer su propia interpretación y debía conformarse con la línea argumental producida por el encargado del guion. Abell Seddon, del museo de historia natural de Birmingham, incluso calificó 


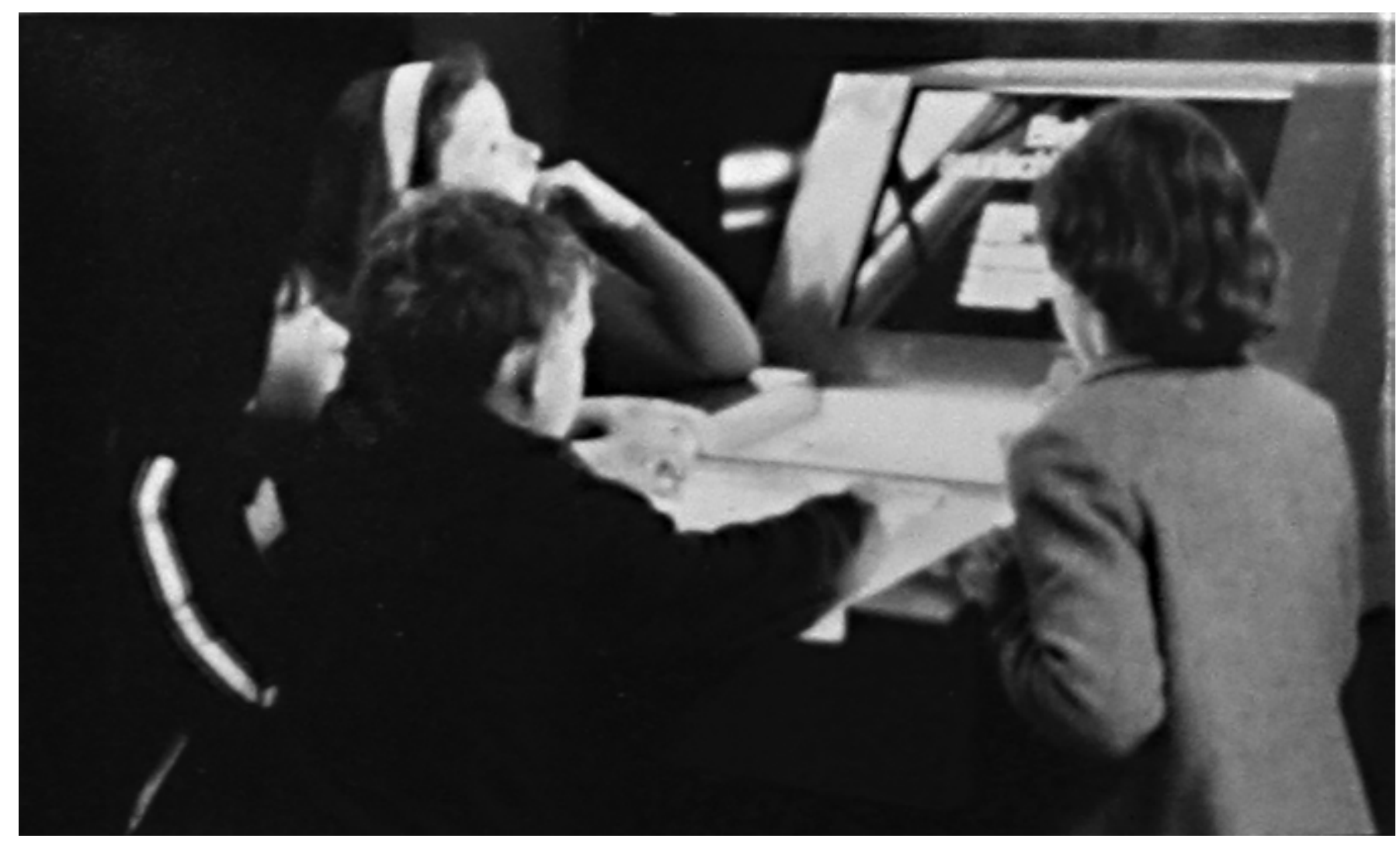

FIGURA 1. Uno de los módulos interactivos instalados en la exposición Human Biology inaugurada en mayo de 1977 en el Natural History Museum (NHM) de Londres. Se trata de la primera exposición del nuevo programa de exposiciones (NPE). Estas exposiciones ya no se basaban sólo en la reunión de colecciones, sino que hacían explícito el mensaje mediante nuevas estrategias comunicativas (Fotografía: Autor desconocido, 1977; cortesía: Natural History Museum [NHM], Reino Unido).

el enfoque propuesto por Miles como propagandístico, coercitivo y con un propósito limitado de antemano. En su opinión, una exposición regida por un guion y estructurada a tal extremo "restringía la libertad del visitante para explorar y descubrir" los elementos que decidiera y como lo decidiera (Seddon 1979).

Los protagonistas del nuevo discurso expositivo serían los modelos y los módulos interactivos (Figura 1). Los críticos calificaban a Human Biology de "patética" (Alt 1979:2): "un proyecto que cayó en las manos del mal gusto de los divulgadores" (Doughty 1978:56). Un disgustado guía voluntario del NHM escribió que el NPE "alcanzó su máxima expresión del mal gusto con la atmósfera de discoteca/parque de diversiones del Human Biology Hall" (Anón. 1981:438). Ante todas esas críticas, los creadores del NPE justificaban las decisiones que tomaron para Human Biology con el argumento de que se trataba de "un experimento para señalar el camino a seguir" o, por qué no, del "preludio de una revolución" (Miles 1986:231; Perks 2012:87). Estas ideas de renovación se materializaron por medio tanto del tema, el propósito y el nombre mismo de la exposición como de las estrategias expositivas, y, por supuesto, de la orientación de los contenidos seleccionados, aspectos que desarroIlo a continuación.

\section{De la historia natural a la "biología moderna"}

La elección de la biología humana como tema de la primera exposición del NPE resultó sorpresiva, ya que se apartaba notablemente de lo que hasta entonces se había mostrado en las galerías. ${ }^{8}$ La decisión respondía tanto a la necesidad de despertar la curiosidad de los visitantes como al deseo de generar una sensación de innovación y entusiasmo por comunicar cuestiones de la ciencia contemporánea a nuevos públicos (NHM 1974a). En una entrevista reciente, Brian Rosen, uno de los científicos participantes en las especificaciones de los contenidos de la exposición piloto, explicó que debía ser algo llamativo que impresionara a los visitantes y garantizara la cobertura de los medios de comunicación (Rosen 2010).

Sin embargo, la razón de que el tema del ser humano fuera el elegido para esta primera exposición no únicamente respondió al interés por cautivar a los visitantes y a

\footnotetext{
${ }^{8}$ Las exposiciones tradicionales, previas al NPE, no se estructuraban temáticamente, sino, más bien, las galerías se categorizaban taxonómicamente. Los curadores diseñaban las exposiciones con base en las líneas de investigación desarrolladas en el museo. De allí que Human Biology haya tenido que coexistir con las galerías de dinosaurios, mamíferos, ballenas, fósiles, minerales/meteoritos, peces y reptiles, insectos, aves y botánica (Alt 1980; Griggs 1990).
} 
los medios: en mi opinión, un par de circunstancias más influyó en esa decisión. En primer lugar, permitía apartarse de las convenciones que Miles le cuestionaba a la museología tradicional, especialmente, su dependencia de las colecciones (Miles 1979; Griggs 1990). En segundo lugar, dada la perspectiva desde la que se abordó, la exposición se diferenciaba de otras muestras sobre el ser humano desarrolladas en otros museos importantes. En los Estados Unidos, por ejemplo, al momento de la inauguración de Human Biology había exposiciones importantes que recurrieron al tema del ser humano para alejarse de los objetos y que seguían una metodología similar a la transformación. ${ }^{9}$ En ellas el tema se trataba desde una perspectiva organísmica y funcionalista en la que la fisiología experimental, la antropología física y la anatomía comparativa jugaban un papel fundamental. Ninguna prestaba mucha atención al discurso de la biología posterior a la Segunda Guerra Mundial: experimental, interdisciplinario y con grandes influencias de la ingeniería y las ciencias de la información. ${ }^{10}$

En cambio, Human Biology, más que destacar la perspectiva anatómica o antropológica de la biología humana, la conectaba con la cibernética, las neurociencias, la psicología cognitiva e, incluso, con la inteligencia artificial (Corral 2015). Basado en la teoría de sistemas, el paradigma de la biología que ofrecía la exposición, en el que el organismo, el cuerpo y la naturaleza habían mutado en sistemas cibernéticos y de control, puede explicarse a partir del perfil de los científicos externos consultados por Miles. Por ejemplo, Young, Gregory y Taylor investigaban la posibilidad de crear máquinas inteligentes capaces de llevar a cabo las mismas funciones que el cerebro. Hobsbaum y Versey eran especialistas en psicología cognitiva y sus investigaciones se centraban, en buena medida, en la relación de la percepción y el conocimiento adquirido mediante la experiencia con el procesamiento de la información (cognición). Jonckheere, por su parte, estaba más interesado en la psicología de la percepción, y buscaba construir modelos estadísticos que explicaran los modelos conceptuales de la mente (Corral 2015:125-130). Esta forma, relativamente nueva, de conceptualizar y estudiar al ser humano, distaba mucho de la aproximación tradicional, que era la antropología física.

El nuevo marco conceptual de las ciencias de la vida que Miles incorporó en los contenidos de Human Biology determinó, asimismo, innovadoras formas de representación. Las imágenes de robots y computadoras, los

\footnotetext{
${ }^{9}$ Una fue Biology of Man, inaugurada en 1961 en el Museo Americano de Historia Natural de Nueva York, pero que en 1977 aún seguía en exhibición (Parr 1959). Otras dos fueron Man in his Environment y The Urban Habitat, en el Museo Field de Chicago y en el Museo Público de Milwaukee respectivamente, ambas, inauguradas en 1976 (Miles 1976:4).

${ }^{10}$ Sobre este enfoque de la biología véanse Galison (1994), Kay (1995) y De Chadarevian (2002).
}

diagramas de flujo propios de la ingeniería y la cibernética, las metáforas básicas del balance y el equilibrio como retroalimentación negativa y otros mecanismos de control, así como los dispositivos interactivos y demás tecnologías educativas, dieron forma a su discurso visual y narrativo. El análisis de la exposición permitirá demostrar esta aseveración.

La exposición abarcaba tres aspectos de la biología humana: crecimiento, control de las acciones y procesos de aprendizaje; estaba formada por los diez pabellones (Figura 2), conectados por una línea argumental que daba sentido a todos los conceptos (Miles y Tout 1978:37).

\begin{tabular}{|c|l|}
\hline PABELLóN & \multicolumn{1}{|c|}{ TíTULO DE SECCIÓN } \\
\hline A & Células \\
\hline B & Crecimiento \\
\hline C & Movimiento \\
\hline D & Controlando tus acciones \\
\hline E & Homeostasis - tu vida en equilibrio \\
\hline F & Hormonas - mensajeros en la sangre \\
\hline G & Hormonas y nervios \\
\hline H & Experiencia de vida - aprendizaje y memoria \\
\hline I & Percepción - entendiendo nuestro mundo \\
\hline J & ¿Cómo llegamos a entender nuestro mundo? \\
\hline
\end{tabular}

FIGURA 2. Pabellones que formaban parte de la estructura temática de la exposición Human Biology (Tabla: Gustavo Corral Guillé, 2017).

Se reemplazaron las vitrinas tradicionales del NHM por audiovisuales y dispositivos electromecánicos. Tal y como explica Claringbull (1974), el guion de la exposición pretendía que el visitante viera nada menos que la historia de sí mismo desde el momento de la concepción, los procesos de desarrollo físico y de crecimiento, así como las hormonas. Según detalla el mismo documento, la historia también comprendía la correlación entre la información recolectada por el cerebro y las acciones humanas como un proceso de retroalimentación continua, $y$, finalmente, diferentes aspectos del aprendizaje, la percepción y las ciencias cognitivas. En cambio, como deja ver la estructura conceptual de los diez pabellones, Human Biology prácticamente no hacía referencia a otras cuestiones de vital importancia en la biología humana, como los sistemas respiratorio, circulatorio, urinario y digestivo, $y$, mucho menos, al inmune. Para entender esa distribución temática tan poco equilibrada debe tenerse en cuenta una de las preocupaciones institucionales que motivó la creación del NPE: proporcionar al público un marco de referencia sobre los temas contemporáneos de la biología y prepararlo para sus desarrollos futuros.

Desde este punto de vista, las colecciones de especímenes "representaban un anacronismo propio del siglo XIX cuando el conocimiento estaba basado en el es- 
tudio descriptivo de las colecciones y no en los experimentos y el análisis de datos" (Corral 2015:150). Esta primera muestra con el nuevo esquema permitió a los diseñadores no sólo experimentar con las tendencias expositivas del momento para potenciar una comunicación más atractiva con el público (Corral 2015): también ofreció la oportunidad de referir el nuevo discurso de la biología que surgió en los Estados Unidos y Europa después de la Segunda Guerra Mundial. Esa representación del cuerpo como sistema de información resultaba natural en lo que Alain Touraine (1969) denominó sociedad postindustrial, que, al final de la década de 1950, había pasado de una economía industrial a una basada en la información. En la ciencia, ese nuevo sistema social y económico que presentaba todo en términos de sistemas computacionales y cibernéticos cobijó el desarrollo de las ciencias cognitivas (que comprendían la inteligencia artificial, las neurociencias y la psicología cognitiva) que, hacia la década de 1970, buscaban adquirir relevancia como un campo interdisciplinario (Gardner 1985). Además, se reinterpretaron en el mismo marco de referencia de la teoría de la información otras dos disciplinas protagonistas de la nueva biología: la genética y la biología celular (Kay 1995).

Los asesores científicos externos de Human Biology fueron formados en el periodo en el que Touraine (1969) localiza el contexto postindustrial, lo que puede explicar la abstracción que hacían de sus disciplinas. La representación naturalista de sus objetos de estudio pasó a convertirse en una simulación, como describiré líneas adelante, debido a esa tendencia a reducir todo en términos de sistemas computacionales y cibernéticos. JeanFrançois Lyotard (1979) advertía, en ese sentido, sobre la existencia de una crisis de representación, idea que más tarde también abordaría Donna Haraway (1991) en lo que Ilamó informática de dominación.

Haraway (1991) cuestionaba la supuesta neutralidad del conocimiento científico, impregnado, como está, de valores e intereses de todo tipo. En este caso, se trataba de un conocimiento ligado al auge de las nuevas tecnologías de la información. Sostenía que, a partir de los sistemas de dirección y control desarrollados en la Guerra Fría, todo tipo de representación de la naturaleza se volvió una simulación: un organismo pasó a ser un componente biótico; el sexo, ingeniería genética; la mente, inteligencia artificial; la fisiología, ingeniería de comunicaciones. Esa visión del mundo como un sistema de redes interconectadas requería, por consiguiente, representaciones más abstractas.

A continuación examinaré detalladamente a qué prácticas expositivas recurrieron los diseñadores de Human Biology para materializar tales representaciones abstractas y los riesgos que éstas conllevaban. El aspecto final resultó condicionado por los intereses y la autoridad de quienes colaboraron en ella, así como por la lógica institucional del museo basada en la modernidad.
Máquinas y cabinas de vuelo. Metáforas en el nuevo discurso del NHM

Desde que comenzó a coordinar los trabajos del NPE, Miles tenía presente que el uso de analogías y metáforas era una de las estrategias de la comunicación científica, que, aplicada al desarrollo expositivo que tenía en mente, le permitiría dar significado al contenido científico y, principalmente, hacer accesibles para el visitante lego los últimos desarrollos de la biología (Miles y Tout 1979; Miles et al. 1982). Miles señalaba al respecto que el proceso de transformación de la información biológica en especificaciones para las exposiciones estaba asociado con "la construcción de analogías o puentes conceptuales, ya sea en forma de modelos gráficos o dispositivos interactivos" (Miles y Alt 1979:161). Es decir, alentó a los científicos-transformadores a emplear las metáforas para comunicar el mensaje que se deseaba transmitir.

Como podrá apreciarse en lo que sigue, esas analogías visuales que se buscó plasmar en la exposición para asignar significado de forma gráfica, Ilamar la atención y hacer accesible la información eran las mismas que utilizaban los científicos cognitivos y los genetistas. Los investigadores de estas disciplinas solían recurrir a metáforas del tipo: el cuerpo es como una máquina, el cerebro es como una computadora o el ADN es como un código de información, para establecer hipótesis sobre el funcionamiento del cerebro y la estructura genética (Kay 1995; Husbands y Holland 2012). Tales figuras eran, por otra parte, un puente entre especialistas de diferentes disciplinas, o bien de una misma, pero de distintos temas.

En el caso de la exposición, tal y como se detallará a continuación, las metáforas más explícitas presentaban al ser humano y su funcionamiento como una computadora, acordes con el perfil de los asesores científicos externos al NHM que colaboraron en la especificación de los contenidos ya mencionados: algunos de ellos describían en sus trabajos de investigación "los patrones de la actividad cerebral como una computadora, y adoptaban un enfoque computacional para explicar las funciones fisiológicas y los mecanismos de aprendizaje" (Corral 2015:154). Por mencionar sólo dos ejemplos, John Zachary Young, quien asesoró en toda la exposición, era especialista en neurobiología, y una de sus principales líneas de investigación era la posibilidad de crear máquinas inteligentes capaces de llevar a cabo las mismas funciones que realiza el cerebro; Richard Langton Gregory, experto en psicología perceptual que colaboró en la sección sobre percepción, defendió a lo largo de su carrera la necesidad de ampliar el conocimiento del funcionamiento del cerebro humano como medio para lograr avances significativos en la inteligencia artificial (Corral 2015:167-168). Conviene entonces revisar de qué tipo de metáforas y analogías se valieron los diseñadores-transformadores para representar y dotar de significado a los aspectos de la biología humana que los asesores exter- 
nos y los científicos consideraban que el público debía aprender. En lo que sigue proporcionaré también algunas posibles explicaciones de los efectos que dichas estrategias producían en la transmisión del conocimiento.

El pabellón D, titulado "Controlando tus acciones", dedicado en su totalidad al cerebro, mostraba, por ejemplo, un modelo que presentaba la corteza cerebral como el centro de control de las acciones del ser humano (Figura 3). Se lo comparaba con la cabina de vuelo de un avión, es decir, el área en la que la tripulación dirige la nave con ayuda de una computadora. La cabina de vuelo contiene el instrumental y los controles que le permiten al piloto regular el movimiento del aparato. De acuerdo con esta metáfora, el cerebro, al igual que la cabina de vuelo, se encargaba de recibir, seleccionar y procesar información para llevar a cabo la mejor acción para sobrevivir. El origen de dicha comparación proviene de la teoría computacional de la mente según la cual ésta computa la información de entrada proveniente del mundo exterior para generar una salida en la forma de un estado mental, o físico, posterior. ${ }^{11}$ Ésta fue la interpretación que se le transmitía a los visitantes cuando observaban este modelo del cerebro.

Brian Rosen fue el científico que especificó la información de este pabellón y, en colaboración con el diseñador Alan Ward; también creó otro módulo, cuyo enfoque se alejaba del "énfasis anatómico y celular para poder mirar el sistema nervioso de una manera más integral" (NHM 1974a:24). Eligieron para ello otra metáfora, en cuyos términos se definía la teoría computacional de la mente: la percepción representa la entrada, la acción realizada es la salida y todo lo que ocurre en el proceso intermedio es parte de un procesamiento de información similar al de las computadoras (Horst 2011). Los dos modelos del cerebro presentados dividían la corteza en tres zonas marcadas con un color diferente:

1. La corteza sensorial, que recolecta la información proveniente de los cinco sentidos.

2. El área de asociación encargada de procesar esta información procedente de las señales de entrada, y responsable, por ejemplo, del entendimiento del lenguaje, las ideas y las decisiones.

3. La corteza motora, que envía las instrucciones de salida a los músculos en forma de señales y a través de los nervios.

Si bien esta comparación probablemente no era tan impactante como la del control de mandos del avión, representaba una nueva muestra de que la analogía del cere-

\footnotetext{
${ }^{11}$ Dicha teoría, propuesta por Hilary Putnam en 1961 y desarrollada por Jerry Fodor en las décadas de 1960 y 1970 (Horst 2011), surgió tras el deseo de averiguar los procesos cerebrales para convertir los estímulos en reacciones, como parte del optimismo descontrolado que generó la inteligencia artificial.
}

bro como máquina procesadora de información era un lugar común en diferentes disciplinas científicas. En el caso de los psicólogos cognitivos, permitía explicar de manera más sencilla el procesamiento de información de un individuo: la recibe por medio de los cinco sentidos y la almacena en la memoria temporal antes de transferir una parte de ella a la memoria a largo plazo y almacenarla en el área de asociación (Rumelhart, Lindsay y Norman 1972).

Habrá que señalar que quizá Rosen no pasó por alto que se abusaba de reduccionismo al concentrar todas las funciones del sistema nervioso (sentir, procesar, responder) en la corteza cerebral. A pesar de ello, con tal de evidenciar visualmente este fenómeno, acordó con su pareja encargada del diseño utilizar esta metáfora. En primer lugar, como se ha apuntado antes, la comunidad científica recurría a ella constantemente. Segundo, había un sentido práctico en su uso: una cuestión de simplicidad, congruente con la intención del NHM de ofrecer exposiciones más didácticas a un público amplio y con pocos conocimientos en biología. El mismo Rosen dejó constancia sobre eso: "Muchos estímulos corporales y muchas de las respuestas del cuerpo son 'manejadas' por otras regiones del cerebro [aparte de la corteza], o incluso completamente fuera del cerebro [...] los siguientes módulos se concentran en la corteza por simplicidad, no porque éste sea el único lugar donde la división tripartita [sensorial, asociativa y motora] se manifieste" (NHM 1974b:25).

El problema con éstas y otras analogías que servían como referente para el público residía en que los creadores de la exposición no establecieron las similitudes y limitaciones entre ellas y el concepto complejo que se buscaba explicar. Como señalan Michel Cloître y Terry Shinn (1985), la inserción de categorías provenientes de otros ámbitos en los campos científicos puede conducir a confusiones cuando la persona no iniciada en la materia intenta resignificarla. Hay, además, algunas metáforas excepcionalmente potentes debido a la riqueza de sus simbolismos, sus vínculos sincrónicos y diacrónicos, y su valor científico y cultural (Kay 1995). En ese sentido, resulta difícil determinar hasta qué punto la que alude a la corteza cerebral como la cabina de vuelo de un avión ofrece una representación clara de aquélla para alguien sin nociones de neurofisiología. En cambio, considerando que a finales de la década de 1970 las computadoras y su funcionamiento resultaban un misterio para la mayor parte de la sociedad, el efecto e impacto de la metáfora podría ser tal que un visitante de Human Biology, al percibir la imagen de la cabina de vuelo, la interpretara no como una representación del cerebro sino como el cerebro. Con la intención de proporcionar estrategias atractivas para representar y hacer visibles conceptos tan abstractos como la percepción y las conexiones neuronales, los diseñadores emplearon imágenes muy sofisticadas y muy hermosas, pero que podían disipar el verdadero significado del fenómeno biológico. De modo que, aunque 


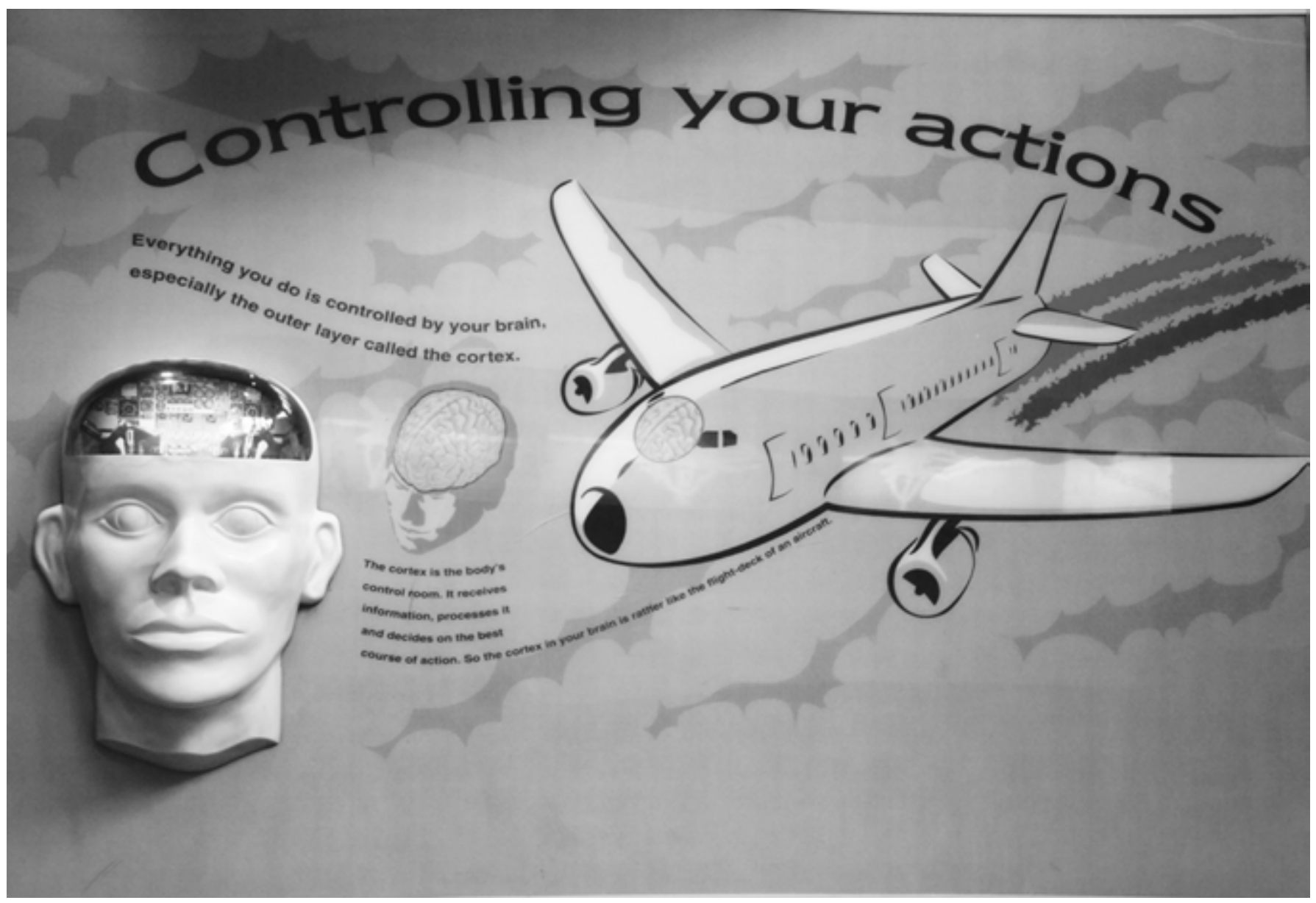

FIGURA 3. Modelo de Human Biology que representaba al cerebro humano como la cabina de vuelo de un avión (Fotografía: Gustavo Corral Guillé, 2013; cortesía: Natural History Museum [NHM], Reino Unido).

las analogías hayan resultado fascinantes e innovadoras en aquel momento, lo que el público extraía de ellas no se compadecía con el significado preciso del fenómeno biológico ilustrado (Corral 2015:158).

Sería un error limitar el uso de analogías y metáforas a su valor práctico como estrategia para visualizar fenómenos biológicos complejos y abstractos. Otro efecto de su uso fue que en la galería se generó una iconografía explícita de lo que, en ese momento, se consideraba la ciencia moderna y, en concreto, la nueva biología, en la que, como ya anoté antes, destacaban las ciencias cognitivas. No debe sorprender, entonces, que Human Biology aludiera a los robots y las computadoras, pues constituían una prueba de que las galerías del NHM mostraban a los visitantes imágenes de la ciencia experimental e interdisciplinaria, la cual resultaba familiar al público. Hacia la década de 1970 las computadoras, los robots y la inteligencia artificial se hallaban en todo tipo de expresiones de la cultura popular, como el cine y la literatura. ${ }^{12}$ Cons-

\footnotetext{
${ }^{12}$ Sirva de ejemplo el Cognitive Science Movie Index (CSMI, Department of Cognitive Science, University of California), EE. UU., que no es una lista exhaustiva, pero en ella pueden contarse aproximadamente 30 películas producidas entre 1968 y 1980 (sólo de habla inglesa) (véase CSMI s.f.).
}

tituían símbolos del frenesí futurista y eran iconos de la idea popular de la alta tecnología.

El único espécimen real en los casi $1000 \mathrm{~m}^{2}$ de esta primera exposición era el cerebro y la médula espinal de un ser humano, con el que el visitante podía explorar el funcionamiento del sistema nervioso central (Perks 2012:296). Predominaban, en cambio, los modelos, los dispositivos interactivos y las imágenes propias de la ciencia ficción, que fueron la respuesta de los científicos y diseñadores del NHM para contrarrestar su falta de experiencia en las nuevas perspectivas de la biología. El incremento de los espacios expositivos del museo acentuó la influencia de asesores externos como Young y Gregory, que colaboraron en Human Biology. Tenían gran visibilidad pública y una trayectoria como comunicadores importante: habían escrito libros de divulgación, colaboraban en la prensa general y frecuentaban la radio y la televisión. Pero, a diferencia de esas estrategias de difusión, las galerías del museo eran una buena oportunidad, menos efímera, para promover su trabajo en busca de recursos: ya fuera la legitimación o el apoyo para sus líneas de investigación. Más que ilustrar la historia y la verdadera naturaleza de la biología humana, su reducción en términos de sistemas computacionales y cibernéticos per- 
mitió al museo ilustrar por qué aquélla era emocionante y divertida.

\section{Conclusión}

Human Biology se convirtió en el proyecto piloto del NPE para presentar al público una concepción moderna de la historia natural. El resultado fue una aproximación interdisciplinaria que estructuraba su discurso en términos de las nuevas tecnologías de la información. Combinaba disciplinas consolidadas, como la anatomía y la fisiología, con otras en proceso de legitimación, como las ciencias cognitivas, y la biología y la genética moleculares. Según los modelos teóricos y las técnicas de investigación de esas nuevas disciplinas, los órganos, células y moléculas están unidos por una red de comunicación que intercambia señales y mensajes. Por lo tanto, las metáforas que poblaron la galería eran más propias de la ingeniería que de la biología, y sirvieron, junto con las nuevas técnicas expositivas del NPE, como estrategia no sólo para simplificar la comprensión del contenido sino también para subrayar la dependencia de la ciencia moderna respecto del equipo y del estímulo que le proporciona la alta tecnificación.

Human Biology fue, a pesar de todo, un punto de inflexión crucial en la transformación del NHM. El cambio repentino de las exposiciones basadas en colecciones a las interactivas sin especímenes fue la respuesta de la institución a un debate sobre la identidad de los museos de ciencia en un momento de grave crisis económica. Tal vez la exposición se concibió como un proyecto piloto del NPE, pero la realidad fue que de su éxito dependían muchas de las decisiones para las exposiciones posteriores. El proceso de producción de esta exposición, Ileno de discusiones acaloradas y decisiones en torno de las nuevas prácticas y del modelo tradicional, ayuda, por lo tanto, a comprender la razón para adoptar determinadas estrategias didácticas y epistemológicas, las cuales estaban más acordes con la redefinición de la ciencia experimentada a partir de la segunda mitad del siglo XX, cuando la atención a la relación con el público adquirió una importancia capital.

Más aún, el deseo de los Trustees de renovar la cara pública del museo obedecía al protagonismo que habían adquirido los visitantes en ese periodo, dada la creciente demanda de rendición de cuentas. Esta nueva aproximación de la biología era la elección más adecuada para transformar la identidad del museo, al proporcionarle una función didáctica y recreativa. Por un lado, facilitó el abandono de las colecciones, pues su discurso aludía al presente y al futuro con mayor naturalidad que al pasado. Por el otro, tanto ese enfoque informático-cibernético de los contenidos como la presentación interactiva y audiovisual de Human Biology basada en las tecnologías educativas y los juegos por computadora se articulaban alrededor de la relación hombre-máquina.
La exposición ofrecía, así, una renegociación de la frontera entre hombre y máquina, primeramente, mediante la presencia generalizada de la interactividad y, enseguida, mediante el vocabulario y la taxonomía de sus exposiciones. En conjunto, sus contenidos y técnicas expositivas expresaban la intención de sus creadores de hacer de la galería una celebración de la tecnología de punta y de la innovación. Y, aunque las metáforas a las que he hecho referencia buscaban dotar de significado a determinados conceptos de la nueva biología para facilitar su comprensión, la muestra excluía cualquier contexto, al sustituir las colecciones de objetos por dispositivos totalmente interactivos (Corral 2015:177-184). La información, que, como se ha comentado, estaba estructurada de forma muy cuidadosa, presentaba únicamente los principios básicos del tema en cuestión y no hacía ninguna referencia a la relevancia de esos conceptos en la vida cotidiana del visitante. Así, éste se veía imposibilitado de construir descripciones de la exposición en función de sus necesidades científicas y sociales.

\section{Referencias}

Alt, Michael

1979 "Letter from Alt to unknown addresseed over the critics of Anthony Smith to the Human Biology exhibition", Londres, Archivo del Museo de Historia Natural, número de referencia DFEXH/700/4.

1980 "Four years of visitor surveys at the Natural History Museum (1976-1979)", Londres, Archivo del Museo de Historia Natural, número de referencia DFEXH/701/23/10.

Anón.

1981 "Letter", Nature, 5797 (289):438.

Arnold, Ken

1996 "Presenting science as product or as process: Museums and the making of science", en Susan Pearce (ed.), Exploring Science in Museums, Londres, Althone Press, 53-78.

Bloom, Joel

1992 "Science and technology museums face the future", en

J. Durant (ed.), Museums and the Public Understanding of Science, Londres, Science Museum, 15-20.

Cannon, John

1962 "The new Botanical Exhibition Gallery at the British Museum (Natural History)", Taxon, 11 (8):248-252.

Claringbull, Frank

1974 "New exhibition scheme: aspects of human development", Londres, Archivo del Museo de Historia Natural, número de referencia DFEXH/700/4/1.

Claringbull, Frank y Lawrence Bragg

1965 Crystal structures of minerals, Londres, George Bell \& Sons.

Cloître, Michel y Terry Shinn

1985 "Expository practice. Social, cognitive and epistemological linkage", en T. Shinn y R. Whitley (eds.), Expository science: Forms and Functions of Popularisation, Dordrecht/ Boston/Lancaster, Reidel, 31-60. 
Corral, Gustavo

2015 "Museo de Historia Natural de Londres, 1968-1981. Una perspectiva histórica", tesis de doctorado en historia de la ciencia, Barcelona, Centre d'Història de la Ciència-Universitat Autònoma de Barcelona (CEHIC-UAB).

2016 "El Museo de Historia Natural de Londres y su nuevo programa de exposiciones [NPE] en la década de 1970. Perfilando nuevos objetivos educativos", Boletín de la Real Sociedad Española de Historia Natural, Sección Aula, Museos y Colecciones, 3:5-19.

Chadarevian, Soraya de

2002 Designs for Life: Molecular Biology after World War II, Nueva York, Cambridge University Press.

CSMI

s.f. Cognitive Science Movie Index, Department of Cognitive Science, University of California, EE. UU., documento electrónico disponible en [https://www.indiana.edu/ cogfilms], consultado en abril de 2017.

Doughty, Philip

1978 "'Britain before man'—a review", Museums Journal, 78 (2):54-56.

Fifield, Richard 1977 "Heritage. Birth of Britain", New Scientist, 1075 (76):238.

Galison, Peter

1994 "The ontology of the enemy: Norbert Wiener and the cybernetic vision", Critical Inquiry, 21:228-266.

Gardner, Howard

1985 The Mind's New Science: A History of the Cognitive Revolution, Nueva York, Basic Books.

Gray, Clive

2015 The Politics of Museums, Londres, Palgrave Macmillan.

Greenaway, Frank

1983 "National museums", Museums Journal, 83 (2):7-12.

Griggs, Steven

1990 "Perceptions of traditional and new style exhibitions at the Natural History Museum", ILVS Review, 1 (2):78-90.

Haraway, Donna 1991 Simians, Cyborgs and Women: the Reinvention of Nature, Nueva York, Routledge.

Horst, Steven

2011 "The computational theory of mind", The Stanford Encyclopedia of Philosophy, documento electrónico disponible en [https://plato.stanford.edu/archives/sum2015/entries/ computational-mind], consultado en marzo de 2017.

Husbands, Phil y Owen Holland 2012 "Warren McCulloch and the British cyberneticians", Interdisciplinary Science Reviews, 37 (3):237-253.

Kay, Lily

1995 "Who wrote the book of life? Information and the transformation of molecular biology, 1945-55", Science in Context, 8 (4):609-634.

Lyotard, Jean François

1979 La condition postmoderne: rapport sur le savoir, París, De Minuit.
Macdonald, Sharon (ed.)

1998 The politics of display: Museums, Science, Culture, Londres, Routledge.

Miles, Roger

1976 "Preliminary report on a visit to 18 museums (sensu lato) in North America and Australia, January and February 1976", Londres, Archivo del Museo de Historia Natural, número de referencia DF700/4.

1979 "First draft paper on the origins of the new exhibition scheme", Londres, Archivo del Museo de Historia Natural, número de referencia DFEXH/700/4.

1986 "Lessons in 'Human Biology'", Museum Management and Curatorship, 5 (3):227-240.

Miles, Roger y Alan Tout

1978 "Human biology and the new exhibition scheme in the British Museum (Natural History)", Curator, 21:36-50.

1979 "Outline of a technology for effective science exhibits", en Bassett Matthew (ed.), Curation of palaeontological collections. Special Papers in Palaeontology, Londres, Paleontological Association, (22):209-224.

Miles, Roger y Michael Alt

1979 "British Museum (Natural History): a new approach to the visiting public", Museums Journal, 78 (4):158-162.

Miles, Roger, Michael Alt, David Gosling, Brian Lewis y Alan Tout (comps.)

1982 The Design of Educational Exhibits, Londres, George Allen \& Unwin.

Morris, Peter (ed.)

2010 Science for the Nation: Perspectives on the History of the Science Museum, Londres, Palgrave Macmillan. $\mathrm{NHM}$

1972 "A proposal for a new approach to the visiting public", Londres, Archivo del Museo de Historia Natural, número de referencia NES DR41.

1972b "Trustees Paper 72/9, new exhibition scheme", Londres, Archivo del Museo de Historia Natural, número de referencia DF933/1012.

1973 "Trustees Meeting, 22 de febrero de 1973", Londres, Archivo del Museo de Historia Natural, número de referencia TRU/900/29.

1974a "A new exhibition on aspects of human development", Londres, Archivo del Museo de Historia Natural, número de referencia $\mathrm{EXH} / 700 / 4 / 1$.

1974b "Aspects of human development: Specimen Section of design brief", Londres, Archivo del Museo de Historia Natural, número de referencia DF EXH/700/4/1/10.

1975 "The new exhibition scheme and its pilot project", Londres, Archivo del Museo de Historia Natural, número de referencia $\mathrm{EXH} / 700 / 4 / 1$.

1977a Human Biology-An Exhibition of Ourselves, catálogo de la exposición, Cambridge, Cambridge University Press. 1977b "Opening of the New Paleontology Wing and the Hall of Human Biology on 24 May 1977", Londres, Archivo del Museo de Historia Natural, número de referencia DF700/4/1/37. 
s.f. Images, recurso electrónico [página web] disponible en [https://nhmimages.com/en/page/show_home_page.html], consultado en abril de 2017.

s.f. Natural History Museum Archive Catalogue, documento electrónico disponible en [http://www.nhm.ac.uk/researchcuration/library/archives/catalogue/DServe.exe?dsqServer $=p$ lacid\&dsqIni=Dserve.ini\&dsqApp=Archive \&dsqDb=Persons \&dsqSearch $=$ Code $==\% 27 \mathrm{PX} 1926 \% 27 \& d s q C m d=S h o w . t c l]$, consultado en marzo de 2017.

Parr, Albert

1959 "Ninetieth annual report of the president", Annual Report of the American Museum of Natural History for 1958, Nueva York, American Museum of Natural History.

Pearson, Lynn

1981 The Organization of the Energy Industry, Londres, Palgrave Macmillan.

Pedretti, Erminia

2002 "T. Kuhn meets T. Rex: critical conversations and new directions in science centres and science museums", Studies in Science Education, 37 (1):1-41.

Perks, Sue

2012 "A definition of the principles of Isotype and an investigation into their methods of diffusion and legacy", tesis de doctorado en comunicación visual, Reading, University of Reading.

Rader, Karen y Victoria Cain

2014 Life on Display: Revolutionizing U. S. Museums of Science and Natural History in the Twentieth Century, Chicago, University of Chicago Press.

Rosen, Brian

2010 Comunicación personal, entrevista realizada por Cathcart Brian para el proyecto de historia oral Museum Lives, Centre for Arts and Humanities Research, Natural History Museum, 16 de febrero.

Rumelhart, David, Peter Lindsay y Donald Norman 1972 "A process model for long-term memory", en Endel Tulving y Wayne Donaldson (eds.), Organization of Memory, Nueva York, Academic Press, 197-246.
Seddon, Abell

1979 "An open letter on exhibition in natural history museums",

Newsletter of the Geological Curators Group, 2 (4):200.

Stearn, William

1981 The Natural History Museum at South Kensington: A His-

tory of the British Museum, Londres, Heinemann.

Touraine, Alain

1969 La sociedad post-industrial, Barcelona, Ariel.

Vergo, Peter

1989 The New Museology, Londres, Reaktion Books Ltd.

Whitehead, Peter y Colin Keates

1981 "The British Museum (Natural History)", Londres, Scala/Philip Wilson.

Woolley, Alan

2014 Comunicación personal, 19 de agosto.

\section{Síntesis curricular del/los autor/es}

\section{Gustavo Corral Guillé}

Investigador independiente, México

gustavo.corral@gmail.com

Maestro en ciencias de la computación (Universidad Nacional Autónoma de México [UNAM]) y doctor en historia de la ciencia (Universidad Autónoma de Barcelona [UAB], España). Sus líneas de investigación se concentran en la historia de los museos de ciencia e historia natural y sus vínculos con la comunicación pública de la ciencia, así como las relaciones entre Estado, economía, sociedad e industria y su impacto en la transformación del discurso expositivo. También está interesado en explorar la ciencia en la esfera pública a lo largo de la historia. Por su tesis doctoral: "El nuevo programa de exposiciones del Museo de Historia Natural de Londres, 1968-1981. Una perspectiva histórica", recibió la distinción de "sobresaliente cum laude".

Postulado/Submitted: 24.09.2016

Aceptado/Accepted: 18.05.2017

Publicado/Published: 15.07.2017 